\title{
Self-induced Pacemaker Mutilation Three Months Post Implant
}

\section{Raffaele Corbisiero*, David Muller RN, Matthew Levy DO and Rebecca Armbuster DO}

Department of Cardiology, Electrophysiology Division, Deborah Heart and Lung Center, USA

Keywords: Attempted suicide; Ideation diagnosis; Pacemaker

\section{Case Presentation}

We present a 58 year old male with past medical history significant for Hypertension, Chronic Paranoid Schizophrenia, Dyslipidemia, Tourette's Syndrome and Heart Block, for which he had recently undergone implantation of a dual chamber Accent DR model 2210 (St Jude Medical, Sylmar CA) with two model 1688 leads (St Jude Medical, Sylmar CA) at a different facility three months prior to this admission. Initially, the patient became distressed at home and notified authorities of suicidal thoughts which consisted of disabling his pacemaker, at which time he was evaluated by emergency medical services.

The patient was brought to a nearby hospital and due to his tenuous mental status as well as hemodynamic compromise, was subsequently admitted. At that time, the patient was noted to be in complete heart block despite his implantable pacemaker. The patient was disorganized in his thoughts and was unable to provide the specific instrument he had used to inflict the damage. He was treated in the ICU where he been restrained for several days as to prevent further self-inflicted harm while being maintained on intravenous Isoproterenol for heart rate support.

At that time, the patient was transferred to our facility for further treatment and management of his pacemaker. Upon our evaluation, the patient was noted to have a series of lesions on the left anterior chest wall, mid-clavicular region overlying his pacemaker pocket. Three traumatic bruises were noted (Figure 1). Two discrete sites of superficial trauma were located just beneath the original, well-healed incision, with the lateral site being somewhat larger. There was also one additional site of trauma inferior to the above which appeared less healed. The remainder of the skin in the region was intact, with mild erythema in a triangular shape noted with the original device incision serving as the apex. No open skin was noted at this time, nor was there exposure of any device components.

Multiple attempts at device interrogation proved unsuccessful, thus the decision was made to replace the device. Due to the patient's tenuous, agitated, and at times aggressive psychiatric state with residual suicidal ideation, it was decided that the procedure should be performed under general anesthesia to better protect the patient's airway. Broad Spectrum Intravenous antibiotics were administered as blood cultures from the transferring facility were still pending. We obtained a CBC which demonstrated normal WBC, coagulation and repeat blood cultures. Due to the patient's unstable mental status and agitation compounded with his unstable hemodynamic condition, it was decided a temporary pacemaker would not be tolerated. After discussions with the patient's power of attorney, it was decided to replace the generator and or leads as needed.

After local anesthetic infiltration, an incision was made over the previous scar. No purulent discharge was noted in the pocket and additional wound cultures were obtained. The device was retrieved and upon visual inspection, a total of 6 indentations of a well-circumscribed nature were noted on the anterior aspect of the can (Figure 2). Two of the six indentations penetrated the titanium case and one caused a concave protrusion on the deep posterior aspect of the can, almost penetrating both the front and back of the titanium casing. The pulse generator was then disconnected from the can.

Upon inspection of the leads, no apparent damage was visually detected. Checking lead integrity through the pacing system analyzer revealed an electrically compromised right ventricular lead. The atrial lead was intact and provided adequate pacing, sensing, and impedance. Subsequently the right ventricular lead was extracted and a new $1888 \mathrm{~T}$ lead (St Jude Medical Sylmar, CA) was placed into the right ventricular outflow tract. To discourage future attempts to damage the device, the new pulse generator was placed in a deep sub-pectoral pocket and the incision was closed (Figure 3).

The manufacturer's analysis reported puncture holes noted on the device casing. The superior penetration was noted on top of the battery (Figure 4). Excessive body fluids were noted inside of the device casing and on the circuitry (Figure 5). Electrical tests were performed and indicated that the pacemaker had no output and no telemetry was possible with a $500 \mathrm{ohm}$ load at $42^{\circ} \mathrm{C}$. Additional attempts to measure output using an oscilloscope also demonstrated no output. It was felt these failures were due to excessive body fluids that caused shorting to the device circuitry. The extracted lead suffered a breach of the insulation.

\section{Discussion}

Blunt trauma as a mode of pacemaker failure has been previously

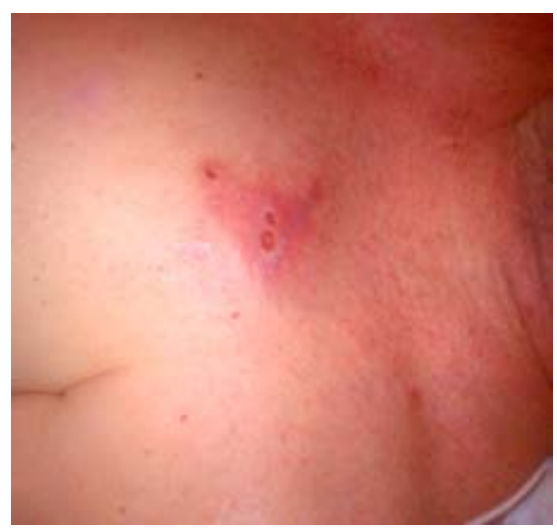

Figure 1: Admission

*Corresponding author: Raffaele Corbisiero, Department of Cardiology, Electrophysiology Division, Deborah Heart and Lung Center, 200 Trenton Road Browns Mills, NJ 08015, USA, Tel: (609) 893-6611; E-mail: corbisieror@deborah.org

Received February 05, 2014; Accepted March 18, 2014; Published March 25, 2014

Citation: Corbisiero R, David Muller RN, Matthew Levy DO, Rebecca Armbuster DO (2014) Self-induced Pacemaker Mutilation Three Months Post Implant. J Cardiovasc Dis Diagn 2: 148. doi:10.4172/2329-9517.1000148

Copyright: @ 2014 Corbisiero R, et al. This is an open-access article distributed under the terms of the Creative Commons Attribution License, which permits unrestricted use, distribution, and reproduction in any medium, provided the original author and source are credited. 
Citation: Corbisiero R, David Muller RN, Matthew Levy DO, Rebecca Armbuster DO (2014) Self-induced Pacemaker Mutilation Three Months Post Implant. J Cardiovasc Dis Diagn 2: 148. doi:10.4172/2329-9517.1000148

reported in the literature and is frequently associated with trauma to the leads at or near the location of the pocket. The mode of trauma ranges from deceleration injuries to blunt trauma with a baseball bat [1-3]. Most common in clinical practice, external traumatic insult to implanted devices is of insufficient force to cause any permanent damage to device function.

Self inflicted causes of device failure are somewhat rare in occurrence, with the exclusion of Twiddler's syndrome first described

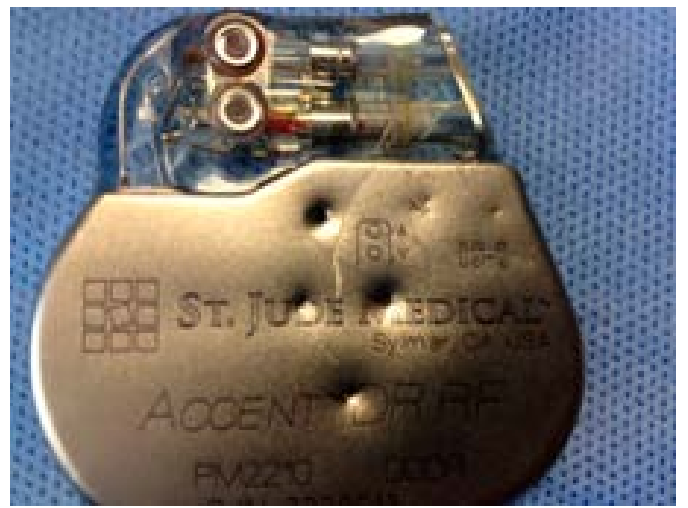

Figure 2: Front view of can post explant. Arrows indicate penetration of titanium Pulse generator case.

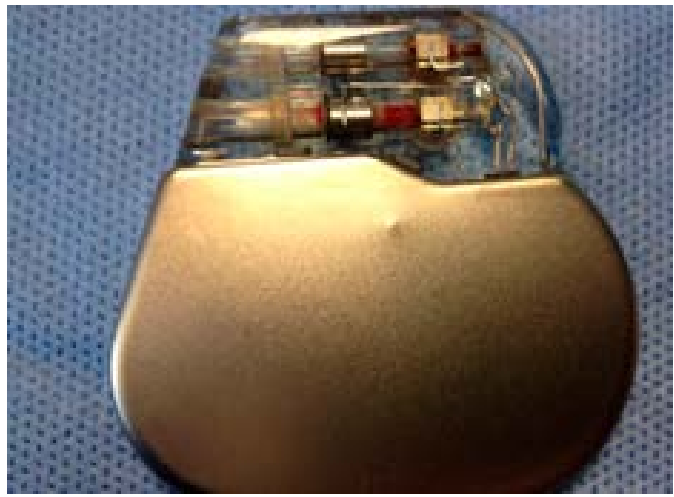

Figure 3: Rear view of can post explant Arrow indicates protrusion from impact through pulse generator.

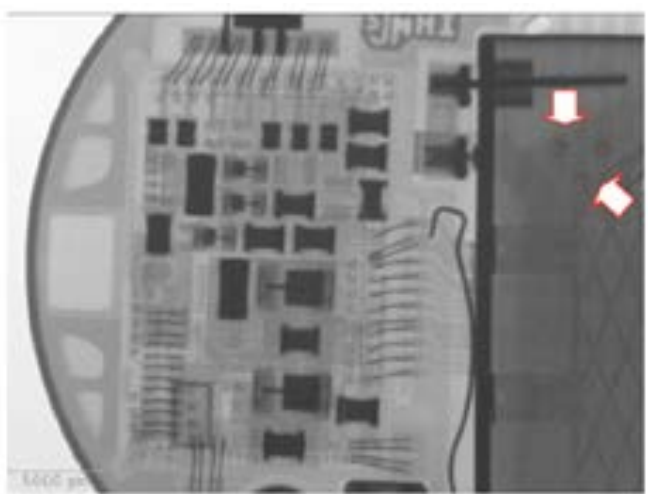

Figure 4: X-Ray of can post implant. Arrows indicate punctures on top of Battery.

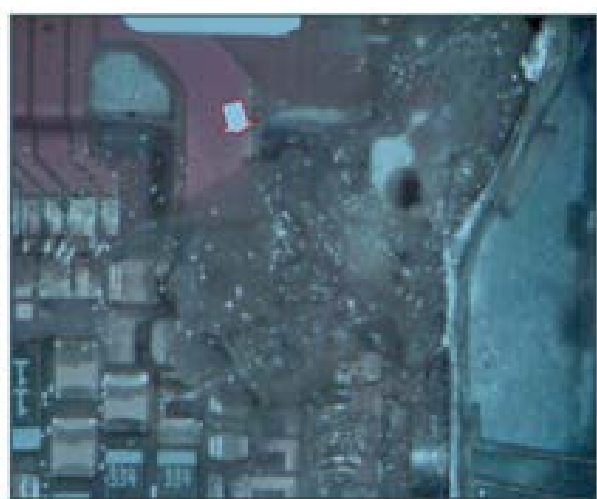

Figure 5: Opened can with body fluids inside of titanium case and noted on the hybrid.

by Bayliss et al. [4]. The body of published literature on self inflicted pacemaker trauma is very limited [5-9]. Given the increasing number of patients receiving implantable cardiac devices, the impact of receiving an implantable device on a patient with an already diagnosed psychiatric condition should be carefully evaluated at the time of implant. The incidence of Schizophrenia has been reported to range from 7.7 to 43 per 100,000 [10-12]. However, diagnosis of freedom from psychiatric disorders at the time of implant does not preclude patients from this type of event from reoccurring. Nonetheless, knowing the diagnosis or risk factors of the disease earlier may help with placement of these devices, for example- deep pectoral placement as an alternative. Some studies have shown late-onset paranoid ideation as first onset estimated to be between $4 \%-6 \%$, however most are concomitantly diagnosed with dementia $[13,14]$. Patients with a first diagnosis at 40 years old have been estimated at $23.5 \%$ with a 1 year prevalence rate of $0.6 \%$ until 64 years of age [15]. After 60 years of age, first admission schizophrenialike psychosis increases by $11 \%$ with each 5 year increase in age [16]. These factors suggest that during the subsequent follow-up years, psychological screening should be repeated annually along with device interrogation to help us intervene and mitigate these events whenever possible [17].

Patients with implantable devices face a myriad of psychological considerations which should be continually monitored. These include the presence of a foreign object implanted in the body, concerns over lead or device malfunction, recalls, battery depletion as well as a continued reminder of their disease state. Although these fears may only affect a small subset of our patient population, care to identify these patients early is paramount. The psychological impact of implantable devices has been underscored over the years with some emphasis on inappropriate high voltage therapy delivered through implantable defibrillators [18-20]. This report underscores the importance of continued psychological evaluation of all device patients including low voltage implantable devices.

\section{References}

1. Kronzon I, Mehta SS (1974) Broken pacemaker wire in multiple trauma: a case report. J Trauma 14: 82-84.

2. Grieco JG, Scanlon PJ, Pifarré R (1989) Pacing lead fracture after a deceleration injury. Ann Thorac Surg 47: 453-454.

3. Brown KR, Carter W Jr, Lombardi GE (1991) Blunt trauma-induced pacemaker failure. Ann Emerg Med 20: 905-907.

4. Bayliss CE, Beanlands DS, Baird RJ (1968) The pacemaker-twiddler's syndrome: a new complication of implantable transvenous pacemakers. Can Med Assoc J 99: 371-373. 
Citation: Corbisiero R, David Muller RN, Matthew Levy DO, Rebecca Armbuster DO (2014) Self-induced Pacemaker Mutilation Three Months Post Implant. J Cardiovasc Dis Diagn 2: 148. doi:10.4172/2329-9517.1000148

Page 3 of 3

5. Stanford W, Coylel FL, Dooley BN, Hood RH (1968)Transvenous Pacemaker Failure: Migration of Catheter Lead by Patient Manipulation. Ann Thorac Surg 5: 162.

6. Meyer JA, Fruehan CT, Delmonico JE Jr (1974) The pacemaker twiddler's syndrome. A further note. J Thorac Cardiovasc Surg 67: 903-907.

7. Rosenthal R, Crisafi BR, Coomaraswamy RP (1980) Manual extraction of a permanent pacemaker: an attempted suicide. Pacing Clin Electrophysiol 3: 229-231.

8. Bordier P, Robert F (2004) Suicide by self-removal of a pacemaker. Am J Forensic Med Pathol 25: 78-79.

9. Simon AB, Kleinman P, Janz N (1980) Suicide attempt by pacemaker system abuse: a case report with comments on the psychological adaptation of pacemaker patients. Pacing ClinElectrophysiol 3: 224-228.

10. Mc Grath J, Saha S, Welham J, El Saadi O, MacCauley C, et al. (2004) A systematic review of the incidence of schizophrenia: the distribution of rates and the influence of sex, urbanicity, migrant status and methodology. BMC Med 2: 13.

11. Mc Grath JJ (2003) Invited commentary: Gaining traction on the epidemiologic landscape of schizophrenia. Am J Epidemiol 158: 301-304.

12. Sartorius N, Jablensky A, Korten A, Ernberg G, Anker M, et al. (1986) Early manifestations and first-contact incidence of schizophrenia in different cultures: A preliminary report on the initial evaluation phase of the WHO Collaborative
Study on Determinants of Outcome of Severe Mental Disorders. Psychol Med 16: $909-928$

13. Christenson R, Blazer D (1984) Epidemiology of persecutory ideation in an elderly population in the community. Am J Psychiatry 141: 1088-1091.

14. Forsell Y, Henderson AS (1998) Epidemiology of paranoid symptoms in an elderly population. Br J Psychiatry 172: 429-432.

15. Harris MJ, Jeste DV (1988) Late-onset schizophrenia: an overview. Schizophr Bull 14: 39-55

16. Keith SJ, Regier DA, Rae DS: Schizophrenic disorders in Psychiatric Disorders in America, (eds.) Robins LN, Regier DA, Free Press: New York, USA.

17. Vanos J, Howard R, Takei N, Murray R (1995) Increasing age is a risk factor for psychosis in the elderly. Soc Psychiatry Psychiatr Epidemiol 30: 161-164

18. Lüderitz B, Jung W, Deister A, Marneros A, Manz M (1993) Patient acceptance of the implantable cardioverter defibrillator in ventricular tachyarrhythmias. Pacing Clin Electrophysiol 16: 1815-1821.

19. Schron EB, Exner DV, Yao Q(2002) Improving Quality ofLife After Cardiogenic Shock: Do More Revascularization. Circulation 105:589-594.

20. Sweeney MO, Wathen MS, Volosin K, Abdalla I, DeGroot PJ, et al. (2005) Appropriate and Inappropriate Ventricular Therapies, Quality of Life, and Mortality Among Primary and Secondary Prevention Implantable Cardioverter Defibrillator Patients Circulation 111: 2898-2905 